\title{
Criptococosis pulmonar en un paciente con colitis ulcerativa tratado con prednisolona y azatioprina
}

\section{Pulmonary cryptococcosis in a patient with ulcerative colitis treated with prednisolone and azathioprine}

Robin Prieto-Ortiz, ${ }^{*}$ 을 Gustavo Reyes, ${ }^{1}$ Germán Carvajal, ${ }^{1}$ Edgar Figueredo. ${ }^{2}$

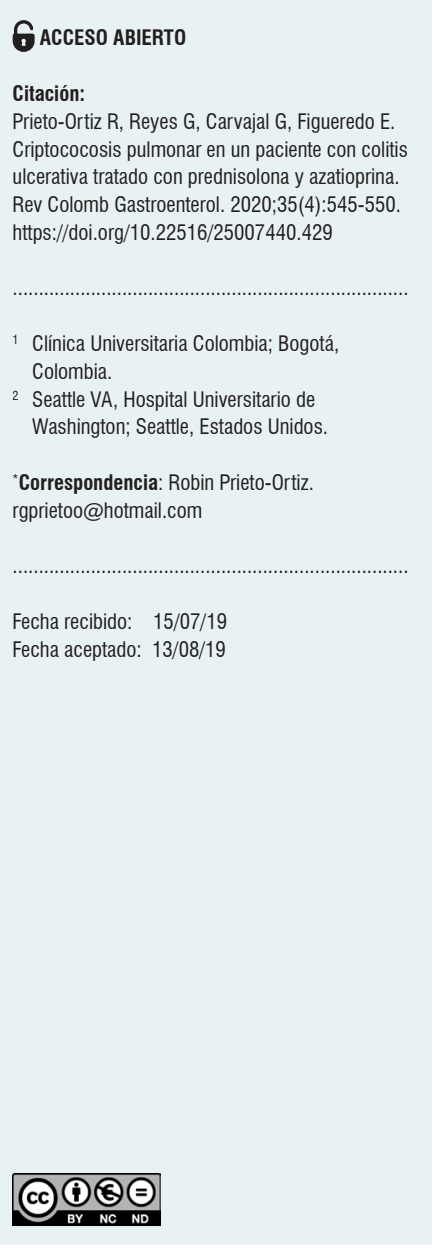

\section{Resumen}

La enfermedad inflamatoria intestinal comprende la enfermedad de Crohn (EC) y la colitis ulcerativa (CU). Esta última es una patología crónica caracterizada por una inflamación difusa de la mucosa colónica, que afecta el recto y se extiende de forma proximal. Su curso clínico es intermitente, con exacerbaciones y remisiones. Su tratamiento, por lo general, es farmacológico, con corticoides, inmunomoduladores e inhibidor del factor de necrosis tumoral (anti tumor necrosis factor, TNF), los cuales causan un estado de inmunosupresión en el paciente, que puede asociarse en algunos casos a infecciones oportunistas. En la literatura se describe la aparición de la criptococosis pulmonar en pacientes con infección por el virus de inmunodeficiencia humana $(\mathrm{VIH})$. En otros casos se asocia al tratamiento farmacológico de pacientes con EC, así como con otras infecciones oportunistas, tales como la tuberculosis y el herpes. Presentamos uno de los primeros casos de criptococosis pulmonar en un paciente con diagnóstico de colitis ulcerativa, quien recibió tratamiento escalonado con salicilatos, inmunomoduladores y terapia biológica. La infección fue documentada clínica, radiológica e histológicamente. El paciente recibió el tratamiento adecuado y presentó una evolución satisfactoria.

\section{Palabras clave}

Enfermedad inflamatoria intestinal, colitis ulcerativa, inmunosupresión, infecciones oportunistas, criptococosis pulmonar.

\begin{abstract}
Inflammatory bowel disease comprises Crohn's disease (CD) and ulcerative colitis (UC), the latter being a chronic disease characterized by diffuse inflammation of the colonic mucosa that affects the rectum and extends proximally. Its clinical course is intermittent with exacerbations and remissions and its treatment is generally pharmacological, with steroids, immunomodulators, and anti-tumor necrosis factor inhibitors (TNF), which cause the patient to be in a state of immunosuppression associated, in some cases, with opportunistic infections. The literature describes the occurrence of pulmonary cryptococcosis in patients with human immunodeficiency virus (HIV) infection, in cases associated with drug treatment of patients with $\mathrm{CD}$, as well as with other opportunistic infections such as tuberculosis and herpes. This is one of the first cases of pulmonary cryptococcosis reported in a patient diagnosed with ulcerative colitis, who received step therapy with salicylates, immunomodulators, and biological therapy. The infection was documented clinically, radiologically, and histologically. The patient received the appropriate treatment and had a satisfactory evolution.
\end{abstract}

\section{Keywords}

Inflammatory bowel disease, Ulcerative colitis, Immunosuppression, Opportunistic infections, Pulmonary cryptococcosis. 


\section{INTRODUCCIÓN}

La colitis ulcerativa (CU) es una enfermedad crónica caracterizada por una inflamación difusa de la mucosa colónica, que afecta el recto y se extiende proximalmente de manera variable, simétrica y circunferencial a todo el colon. El síntoma cardinal es la diarrea sanguinolenta, asociada con urgencia y tenesmo rectal, y su curso clínico es intermitente, con exacerbaciones y remisiones (1). Generalmente, el tratamiento inicial es farmacológico y solo en algunos casos es quirúrgico. El uso de algunos medicamentos, como corticoides, inmunomoduladores y anti factor de necrosis tumoral (tumor necrosis factor, TNF), causa un estado de inmunosupresión en el paciente, que se puede asociar en algunos casos a infecciones oportunistas (2).

Presentamos el caso de un paciente masculino con diagnóstico de $\mathrm{CU}$, quien recibió tratamiento escalonado con salicilatos, inmunomoduladores y terapia biológica, sin lograr un control adecuado de la enfermedad, por lo cual fue remitido a cirugía, y tuvo una correcta evolución posoperatoria. Durante su tratamiento, el paciente presentó criptococosis pulmonar, la cual fue documentada clínica, radiológica e histológicamente. El individuo recibió el tratamiento adecuado y presentó una evolución satisfactoria.

\section{CASO CLÍNICO}

Paciente masculino de 48 años, quien en noviembre de 2011 consultó por primera vez por deposiciones diarreicas con moco y sangre, de 8 meses de evolución, y pérdida de $10 \mathrm{~kg}$ de peso, sin otros antecedentes de importancia. En el examen físico, presentó un leve dolor a la palpación en el marco cólico. Se realizó una colonoscopia con hallazgo de colitis extensa, y se tomaron biopsias que confirmaron el diagnóstico de $\mathrm{CU}$, por lo cual se inició tratamiento con mesalazina $3 \mathrm{~g} /$ vía oral (VO)/día y un ciclo de corticoides (prednisolona) de $40 \mathrm{mg} / \mathrm{VO} /$ día, con mejoría clínica. El paciente fue dado de alta con indicación de continuar la mesalazina de forma permanente, así como los corticoides, con una posterior disminución gradual y suspensión.

Tres meses después, y luego de la suspensión anticipada y voluntaria de ambos medicamentos, el paciente presentó una recaída. La colonoscopia informó pancolitis ulcerativa, con índice 2 de severidad, según la subclasificación endoscópica de Mayo, y estado E3 S2, de acuerdo con la clasificación de Montreal. Se reinició la mesalazina $3 \mathrm{~g} / \mathrm{VO} /$ día, lo que condujo a la resolución de su sintomatología.

Sin embargo, en octubre de 2013, el paciente presentó una nueva recaída, con un examen coproscópico que evidenció amebiasis, la cual fue tratada con metronidazol más nifuroxazida cada $12 \mathrm{~h}$, por 7 días, sin que se evidenciara una mejoría. Por esto, y considerando una reactivación de la enfermedad, se decidió adicionar azatioprina $150 \mathrm{mg} /$ $\mathrm{VO} /$ día $(2,5 \mathrm{mg} / \mathrm{kg} /$ día $)$ a la mesalazina que el paciente venía tomando, con lo cual se logró una adecuada remisión.

El paciente consultó nuevamente en noviembre de 2014 por presentar deposiciones diarreicas con sangre, malestar general, astenia y adinamia, y pérdida de $4 \mathrm{~kg}$ de peso. Venía tomando mesalazina en $3 \mathrm{~g} / \mathrm{VO} /$ día y azatioprina en $50 \mathrm{mg} / \mathrm{VO} / 8 \mathrm{~h}(2,5 \mathrm{mg} / \mathrm{kg} /$ día $)$. Se efectuó un diagnóstico de cor anémico, hemorragia de las vías digestivas bajas y CU activa corticodependiente, con actividad severa (Montreal S3), y se inició un pulso de corticoides (prednisolona $40 \mathrm{mg} /$ día). Además, se le transfundieron 2 unidades de glóbulos rojos empaquetados y, posteriormente, hierro sacarosa.

En un nuevo control endoscópico, se encontró pancolitis ulcerativa y pseudopólipos, que en comparación con la colonoscopia previa, evidenció una progresión de la enfermedad, por lo que se contempló el inicio de una terapia biológica. Se solicitaron pruebas de tuberculina (Purified protein derivative, $\mathrm{PPD}$ ), serología para hepatitis $\mathrm{A}, \mathrm{B}, \mathrm{C}$ y una prueba de VIH. Se realizó además una radiografía de tórax, en la que se observó la presencia de un nódulo en el lóbulo superior izquierdo y otro en el lóbulo inferior, de 6 $\mathrm{mm}$ cada uno (Figura 1). La tomografía computadorizada de tórax confirmó dichos hallazgos (Figura 2).

Ante la sospecha de un tuberculoma, se realizó una biopsia en cuña del nódulo del lóbulo inferior, con una patología que reportó un parénquima pulmonar con enfermedad granulomatosa crónica caseificante, compatible con criptococosis, y negativo para bacilos acidorresistentes (BAAR) (Figura 3). Los cultivos de hongos confirmaron un moderado crecimiento de Cryptococcus neoformans, mientras que la PPD negativa descartó la tuberculosis latente.

Frente al diagnóstico de criptococosis pulmonar sin compromiso sistémico, el servicio de infectología indicó el comienzo del tratamiento con fluconazol en $400 \mathrm{mg} / \mathrm{VO} /$ día y contraindicó empezar la terapia biológica durante, por lo menos, las ocho semanas iniciales del tratamiento con fluconazol. Además, se indicó un control sérico mensual de criptolátex durante el primer año, el cual fue negativo.

Dos meses después de haber iniciado el tratamiento con fluconazol, se añadió adalimumab, además de la mesalazina y la azatioprina que el paciente venía recibiendo. Inicialmente, este presentó una adecuada respuesta, pero luego de ocho meses reingresó por una nueva recaída. Se consideró entonces una reactivación de la CU por evolución de la enfermedad (estado S3, según la escala de severidad de Montreal) y una colitis severa, de acuerdo con los criterios de Truelove y Witts.

Sin embargo, ante la respuesta no adecuada al adalimumab se inició tratamiento con infliximab, con lo cual el paciente presentó una mejoría inicial de su sintomatología. 


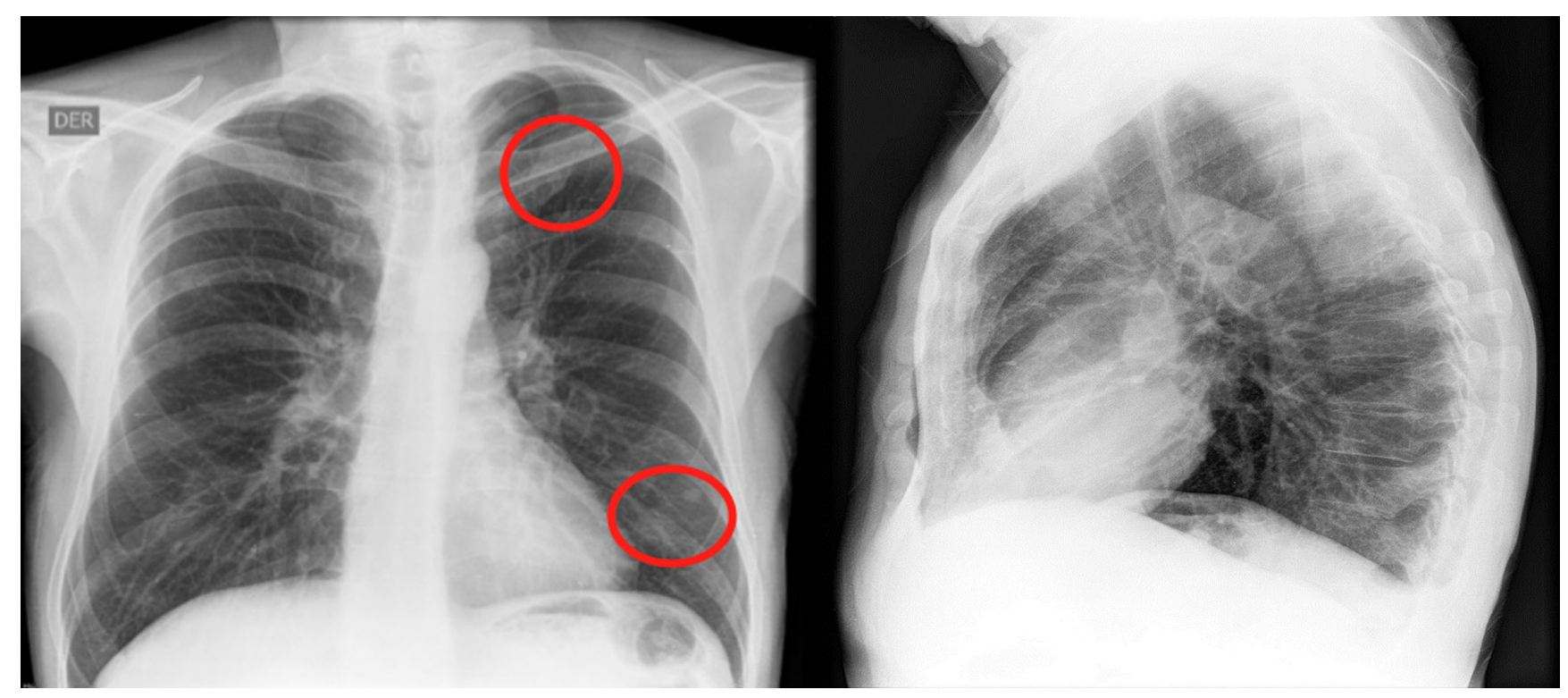

Figura 1. En la radiografía de tórax se observan opacidades nodulares con densidad de tejidos blandos, proyectadas a nivel de los lóbulos superior e inferior izquierdo. Fuente: archivo de los autores.

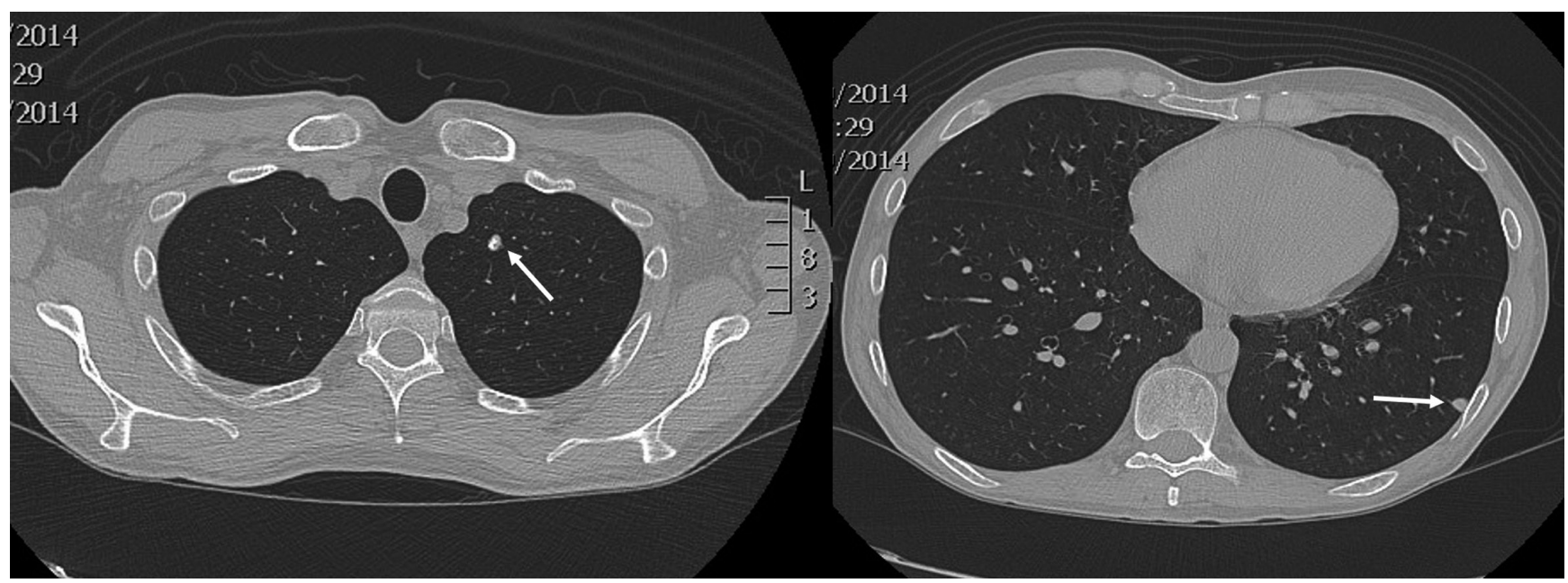

Figura 2. Se observa un nódulo pulmonar con calcificaciones gruesas en el segmento apicoposterior del lóbulo superior izquierdo, de 6 mm de diámetro, y otra imagen nodular subpleural de $6 \mathrm{~mm}$ de diámetro, en el segmento superior del lóbulo inferior izquierdo, con densidad de tejidos blandos. Fuente: archivo de los autores.

Pese a ello, en octubre de 2015 tuvo otra recaída, razón por la que se consideró una falla en la respuesta al tratamiento biológico y se determinó un tratamiento quirúrgico.

Así pues, se realizó un proctocolectomía total, un reservorio ileal en J, una anastomosis ileoanal y una ileostomía de protección. En un segundo tiempo (febrero de 2016), se efectuó el cierre de la ileostomía. En la actualidad, el paciente permanece asintomático, refiere una adecuada calidad de vida y no requiere medicamentos.

\section{DISCUSIÓN}

En las últimas décadas, las infecciones oportunistas, como la tuberculosis y la criptococosis, se han incrementado debido especialmente a la aparición de patologías como el síndrome de inmunodeficiencia adquirida (SIDA), en la que la criptococosis puede ocurrir en el 5 y $10 \%$ de los casos. Estas enfermedades oportunistas también han sido informadas en pacientes sometidos a estados de inmunosu- 


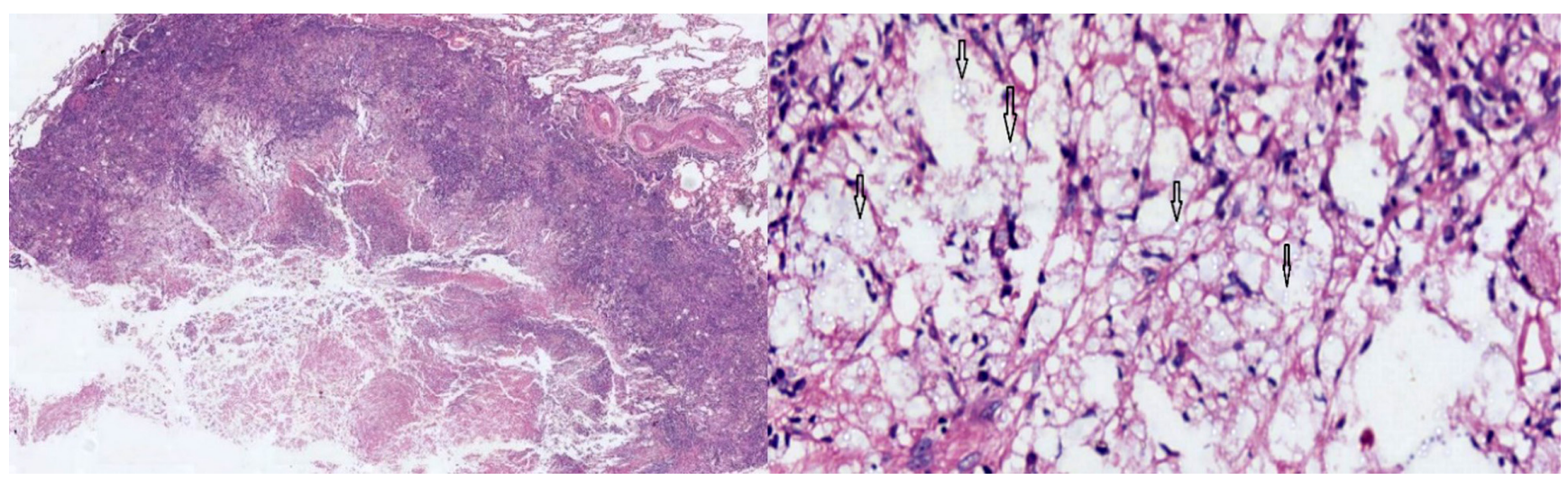

Figura 3. Con coloración de hematoxilina-eosina, se observa el parénquima pulmonar con un granuloma necrotizante, con detritos e histiocitos. A mayor aumento $(10 \mathrm{X})$, se aprecian en detalle las esporas del criptococo, señaladas con flechas. Fuente: archivo de los autores.

presión como parte del tratamiento de trasplante de órganos o en aquellos con enfermedades de tipo inflamatorio; incluso pueden observarse en personas inmunocompetentes. Se estima que a nivel mundial se dan hasta un millón de nuevos casos de criptococosis, con cerca de 650000 muertes por año $(3,4)$.

La criptococosis pulmonar es causada por una levadura encapsulada, que se adquiere por inhalación de esporas del medio ambiente (5). Existen varias especies, pero las más comunes son la del tipo neoformans, que se pueden encontrar en cualquier parte del mundo; la variedad gattii se halla en regiones tropicales y subtropicales (6).

En la mayoría de los casos, se logra determinar la exposición a heces de paloma, aves de corral, perros, gatos u otras esporas del medio ambiente. Sin embargo, hasta en un $15 \%$ de los casos no se identifica la fuente de contacto (7). En el contexto de nuestro paciente, no se documentó exposición alguna.

Los pacientes pueden presentar desde síntomas leves, como cefalea, tos y fiebre, hasta un compromiso sistémico que se manifiesta con escalofríos, hemoptisis y pérdida de peso. En un porcentaje que varía (según diversas publicaciones), en el 17-35\% de los casos, los individuos pueden estar asintomáticos. El diagnóstico se hace frecuentemente de forma incidental, debido al hallazgo radiológico de nódulos pulmonares, los cuales pueden ser solitarios o múltiples, así como de áreas de consolidación o patrón reticulonodular $(8,9)$.

Nuestro paciente no presentó síntomas relacionados con la criptococosis. Por tanto, el diagnóstico fue incidental, a partir del hallazgo inicial de nódulos pulmonares que se confirmaron mediante tomografía. Esta técnica forma parte de los métodos diagnósticos y en ella, usualmente, se observan pocos o múltiples nódulos subpleurales o masas con o sin halo. Además, en un menor número de casos, pueden apreciarse consolidaciones, derrames pleurales o un aumento del tamaño de los ganglios mediastínicos $(10,11)$.
Asimismo, se emplean varias técnicas diagnósticas como la medición sérica del antígeno capsular. Esta tiene la ventaja de ser un método no invasivo, pero del cual se han informado falsos negativos y positivos, razón por la que es necesario ser cautos en su análisis (7). En la actualidad también se utilizan pruebas de biología molecular, las cuales tienen la ventaja de ser rápidas, pero requieren más tiempo de uso para su adecuada evaluación.

Los medios diagnósticos considerados más específicos son el cultivo y la biopsia del tejido; esta última es la más importante y se puede obtener por punción o quirúrgicamente. Las coloraciones empleadas son las de tinta china, Gomory, ácido peryódico de Schiff (Periodic Acid-Schiff, PAS) y mucicarmina (12). En el caso de nuestro paciente, se realizaron cultivo y biopsia, que demostraron la presencia de las esporas del criptococo.

El tratamiento de la criptococosis en pacientes inmunocomprometidos, mediante fluconazol, se inició en la década de los 90 y logró una marcada disminución de la presencia de esta enfermedad (13). Actualmente, en las infecciones pulmonares leves o moderadas, se indica un tratamiento con fluconazol (400 m/día) durante 6-12 meses. En efecto, este fue el tratamiento de nuestro paciente.

Entre tanto, en infecciones graves o con compromiso nervioso central, se usa una inducción con anfotericina $B$ liposomal (3-4 mg/kg/día, intravenosa [IV]) o un complejo lipídico de anfotericina B ( $5 \mathrm{mg} / \mathrm{kg} /$ día, IV) durante 2 semanas, seguido de fluconazol (400 mg/día) por 8 semanas $(14,15)$.

En los últimos años, se ha observado la aparición de la resistencia al fluconazol, especialmente en países orientales. Dicha resistencia aumentó del 5,1 al 22,6\%, en un período de 7 años, según diversos informes. Esto ha ocurrido debido al uso excesivo del medicamento para realizar profilaxis en pacientes inmunocomprometidos o a su 
administración en tratamientos de terapia primaria o de mantenimiento (16).

Son pocos los casos informados de pacientes con enfermedad inflamatoria intestinal y criptococosis. En todos ellos se ha relacionado con la administración de anti TNFa, mientras que en la mayoría de escenarios se ha asociado en pacientes con EC (17). Hasta ahora, no se han informado casos de criptococosis en personas con CU tratadas con corticoides y azatioprina.

\section{CONCLUSIONES}

Consideramos que este es el primer caso informado en la literatura de un paciente con CU tratado con corticoides y azatioprina. El individuo presentó una criptococosis pulmonar, que es una enfermedad oportunista, la cual afecta especialmente a pacientes con SIDA. Sin embargo, también se ha observado en sujetos trasplantados y en aquellos tratados por enfermedades de tipo inflamatorio. Estos pacientes se consideran inmunosuprimidos y en ellos la infección por criptococos puede ser asintomática, por lo que antes del inicio de la terapia biológica se deben realizar los estudios correspondientes.

\section{Conflictos de intereses}

Ninguno.

\section{REFERENCIAS}

1. Hani AC, Galiano MT, Albis R, Márquez JR, Juliao F. Consenso Colombiano de Enfermedad Inflamatoria Intestinal. Rev Col Gastroenterol. 2012;27(Supl. 1):S1-S44.

2. Chen LP, Li J, Huang MF, Chen QS, Xia B. Cryptococcus neoformans infection in ulcerative colitis with immunosuppressants. Inflamm Bowel Dis. 2011;17(9):2023-4. http://doi.org/10.1002/ibd.21619

3. Pappas PG. Cryptococcal infections in non-HIV-infected patients. Trans Am Clin Climatol Assoc. 2013;124:61-79.

4. Ye F, Xie JX, Zeng QS, Chen GQ, Zhong SQ, Zhong NS. Retrospective analysis of 76 immunocompetent patients with primary pulmonary cryptococcosis. Lung. 2012;190(3):339-46. http://doi.org/10.1007/s00408-011-9362-8

5. Goldman DL, Khine H, Abadi J, Lindenberg DJ, Pirofski La, Niang R, Casadevall A. Serologic evidence for Cryptococcus neoformans infection in early childhood. Pediatrics. 2001;107(5):E66. http://doi.org/10.1542/peds.107.5.e66

6. Baddley JW, Dismukes WE. Cryptococcosis. En: Kauffman CA, Pappas PG, Sobel JD, Dismukes WE. Essentials of Clinical Mycology. Nueva York: Springer; 2011. p. 207-226.

7. Zhang Y, Li N, Zhang Y, Li H, Chen X, Wang S, Zhang $\mathrm{X}$, Zhang R, Xu J, Shi J, Yung RC. Clinical analysis of 76 patients pathologically diagnosed with pulmonary cryptococcosis. Eur Respir J. 2012;40(5):1191-200. http://doi.org/10.1183/09031936.00168011

8. Suwatanapongched T, Sangsatra W, Boonsarngsuk V, Watcharananan SP, Incharoen P. Clinical and radiologic manifestations of pulmonary cryptococcosis in immunocompetent patients and their outcomes after treatment. Diagn Interv Radiol. 2013;19(6):438-46. http://doi.org/10.5152/dir.2013.13049

9. Chang YS, Chou KC, Wang PC, Yang HB, Chen $\mathrm{CH}$. Primary pulmonary cryptococcosis presenting as endo- bronchial tumor with left upper lobe collapse. J Chin Med Assoc. 2005;68(1):33-6. http://doi.org/10.1016/S1726-4901(09)70129-7

10. Lindell RM, Hartman TE, Nadrous HF, Ryu JH. Pulmonary cryptococcosis: CT findings in immunocompetent patients. Radiology. 2005;236(1):326-331. http://doi.org/10.1148/radiol.2361040460

11. Yu JQ, Tang KJ, Xu BL, Xie CM, Light RW. Pulmonary cryptococcosis in non-AIDS patients. Braz J Infect Dis. 2012;16(6):531-539. http://doi.org/10.1016/j.bjid.2012.07.004

12. Singh N, Alexander BD, Lortholary O, Dromer F, Gupta KL, John GT, del Busto R, Klintmalm GB, Somani J, Lyon GM, Pursell K, Stosor V, Muñoz P, Limaye AP, Kalil AC, Pruett TL, Garcia-Diaz J, Humar A, Houston S, House AA, Wray D, Orloff S, Dowdy LA, Fisher RA, Heitman J, Wagener MM, Husain S. Pulmonary cryptococcosis in solid organ transplant recipients: clinical relevance of serum cryptococcal antigen. Clin Infect Dis. 2008;46(2):e12-8. http://doi.org/10.1086/524738

13. Rohatgi S, Pirofski LA. Host immunity to Cryptococcus neoformans. Future Microbiol. 2015;10(4):565-581. http://doi.org/10.2217/fmb.14.132

14. Ali T, Kaitha S, Mahmood S, Ftesi A, Stone J, Bronze MS. Clinical use of anti-TNF therapy and increased risk of infections. Drug Healthc Patient Saf. 2013;5:79-99. http://doi.org/10.2147/DHPS.S28801

15. Zhou Q, Hu B, Shao C, Zhou C, Zhang X, Yang D, Li C. A case report of pulmonary cryptococcosis presenting as endobronchial obstruction. J Thorac Dis. 2013;5(4):E170-3. http://doi.org/10.3978/j.issn.2072-1439.2013.08.40

16. Pfaller MA, Diekema DJ, Gibbs DL, Newell VA, Bijie H, Dzierzanowska D, Klimko NN, Letscher-Bru V, Lisalova M, Muehlethaler K, Rennison C, Zaidi M; Global Antifungal 
Surveillance Group. Results from the ARTEMIS DISK Global Antifungal Surveillance Study, 1997 to 2007: 10.5-year analysis of susceptibilities of noncandidal yeast species to fluconazole and voriconazole determined by CLSI standardized disk diffusion testing. J Clin Microbiol. 2009; 47(1):117-23.

http://doi.org/10.1128/JCM.01747-08
17. Fraison JB, Guilpain P, Schiffmann A, Veyrac M, Le Moing V, Rispail P, Le Quellec A. Pulmonary cryptococcosis in a patient with Crohn's disease treated with prednisone, azathioprine and adalimumab: exposure to chicken manure as a source of contamination. J Crohns Colitis. 2013;7(1):e11-4.

http://doi.org/10.1016/j.crohns.2012.04.016 